\title{
Antibiotic resistance in uropathogenic Escherichia coli isolated from urinary tract infections out-patients in Kermanshah
}

Somayeh Jalilian', Abbas Farahani', Parviz Mohajeri ${ }^{2 *}$

${ }^{1}$ Student Research Committee, Kermanshah University of Medical Sciences, ${ }^{2}$ Department of Microbiology, School of Medicine, Kermanshah University of Medical

Sciences, Kermanshah, Iran

Address for the Correspondence: Dr. P. Mohajeri,

Assistant Professor of Medical Bacteriology, Department of Microbiology, Faculty of Medicine, School of Medicine, Kermanshah University of Medical Sciences, Shirudi Shahid Blvd, Daneshgah Street, Postal Code 67148-69914, Kermanshah, Iran.

E-mail: p_mohajeri@yahoo.com

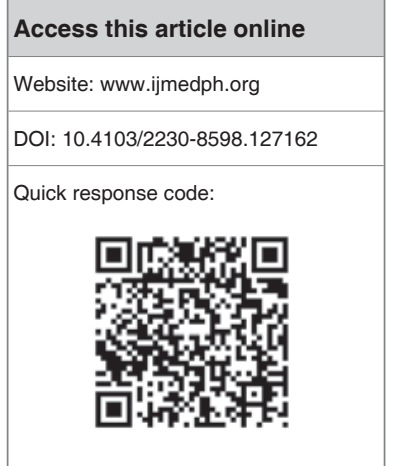

Background: Urinary tract infections (UTIs) are common cause of infections described in out-patient's setting and increase in antibiotic resistance of Escherichia coli, is encountered world-wide. Antibiotic treatment is usually empirical; therefore, this study to provide the knowledge of local resistance pathogen patterns in Kermanshah. Materials and Methods: We conducted a retrospective analysis of all E. coli isolates from urine samples admitted to Kermanshah Central lab between March 2011 and 2012 were included. Antimicrobial resistance was tested by the Kirby-Bauer disk diffusion. Results: This study showed a total of 20,742 samples, 1228 (5.92) were positive for pathogenic bacteria. E. coli were the predominant 801 isolate $(65.2 \%)$. Out of the 13 antibiotics tested for $E$. coli isolates, minimum and maximum resistance were observed to ampicillin (9.4\%) and augmentin (68.6\%). Almost $59-66 \%$ of the uropathogenic $E$. coli strains were resistant to amikacin, co-trimoxazole, tetracycline and cephalotin and nearly half of them were resistant to nalidixic acid and cephalexin. Conclusion: This study confirms that $E$. coli is still the most common uropathogen isolated. Augmentin and amikacin are not as a first choice for treatment of UTI in Kermanshah area. Ampicillin and nitrofurantoin may be considered as a first choice empiric agent in out-patients.

Key words: Antimicrobial resistance, Escherichia coli, urinary tract infections

\section{INTRODUCTION}

Urinary tract infections (UTIs) remain one of the most common bacterial infections in both community and health-care settings. ${ }^{[1]}$

On a global basis, 150 million people are diagnosed with UTI each year and costing in excess of 6 billion dollars in direct health-care costs in the United States. ${ }^{[2]}$ The predominance of Gram-negative species particularly, Escherichia coli, remain the predominant pathogen causing UTI, accounts for $75-90 \%$ of all UTIs in both in-patients and out-patients. It is a known fact that an increase in the antimicrobial resistance among pathogens is a problem encountered world-wide. ${ }^{[3]}$ Most of the cases of UTI are uncomplicated and in the majority of cases, antibiotics are usually given empirically before the laboratory results of urine culture are available. Therefore, area-specific monitoring studies to ensure appropriate therapy, current knowledge of the organisms that cause UTI and their antibiotic susceptibility is mandatory for helping the selection of an effective empirical treatment. The in vitro resistance rate of uropathogenic E. coli strains (UPECs) to the most commonly prescribed antimicrobial agents for treatment of UTI infections vary considerably in different areas of the world-wide. Therefore it is necessary to have updated knowledge of causal bacteria and their susceptibility patterns for proper selection and use of antibiotics as well as for an appropriate prescribing guideline. ${ }^{[4]}$ It is hoped that the information gained from this study would help in the treatment of cases of UTI in our area. Antibiotic susceptibility pattern differences seen between countries and also different medical regions within every country. This motivated us to conduct the current study, as there have been few studies in this respect in our country..$^{[5]}$ The aim of the present study is to determine the current levels of resistance to antibiotics commonly used locally for evidence based therapeutic guidelines and empirical treatment we reviewed susceptibility of out-patient $E$. coli urinary isolates obtained in our laboratory to avoid inter-center deviations in methods and interpretations over a 1 year period. 


\section{MATERIALS AND METHODS}

The study is a retrospective analysis of all E. coli isolates recovered from urine samples admitted to Kermanshah Central lab between March 2011 and 2012 were included. This public clinical laboratory is one of big labs located in west of Iran, where patients across the province visit regularly. All patients with pyuria ( $>10$ white blood cells $/ \mu \mathrm{L})$, acute voiding symptoms and significant bacteriuria $\left(>10^{5}\right.$ colony forming units [CFU]/ml) from a freshly voided mid-stream urine specimen were included in the microbiological analysis. Only one specimen per patient was included in the study.

Antimicrobial susceptibility of isolates was tested by Kirby-Bauer disk diffusion method following the definition of Clinical and Laboratory Standards Institute (CLSI) interpretive breakpoint. ${ }^{[6]}$ A pure culture of the organism, which had been freshly grown on blood agar, was suspended in normal sterile saline and the turbidity of the suspension is adjusted to an equivalent $0.5 \mathrm{McF}$ arland standard..$^{[7]}$

The bacterial suspension was inoculated on Mueller-Hinton agar (Merck) as per guidelines for disc diffusion technique. Antibiotic impregnated disks (MAST Company) were applied to the MuellerHinton agar plates. The plates were incubated aerobically at $37^{\circ} \mathrm{C}$ for 18-24 h and colony counts were expressed in CFU/ $\mathrm{ml}$ of urine. Zone sizes of inhibition were read and interpreted for susceptibility in accordance with the currently recommended CLSI criteria for urinary tract isolates of Enterobacteriaceae. Antibiotic concentrations in the diffusion discs used for antimicrobial susceptibility testing are: amikacin $(30 \mu \mathrm{g})$, amoxicillin $(10 \mu \mathrm{g})$, ceftizoxim $(30 \mu \mathrm{g})$ cephalexin (30 $\mu \mathrm{g})$, chloramphenicol $(30 \mu \mathrm{g})$, co-trimoxazole $(1.25 / 23.75 \mu \mathrm{g})$, gentamicin $(10 \mu \mathrm{g})$, nitrofurantoin $(300 \mu \mathrm{g})$, norfloxacin $(10 \mu \mathrm{g})$, tetracycline $(30 \mu \mathrm{g})$, coamoxicylin $(30 \mu \mathrm{g})$, cephalothin $(30 \mu \mathrm{g})$, amikacin $(30 \mu \mathrm{g})$ (MAST, Merseyside, U.K). Quality control was performed once weekly using test strains E. coli ATCC 25922 , Staphylococcus aureus ATCC 25923 and Pseudomonas aeruginosa ATCC 27853. Data processing and statistical analysis were performed using SPSS software (version 16.0, SPSS, Inc., Chicago, IL). The results were analyzed using the descriptive statistics. The Chi-square and Fisher's exact tests were applied for categorical variables. All statistical tests were two-tailed and $P<0.05$ was considered to be statistically significant. Extended-spectrum B-lactamase (activity) was detected by phenotypic confirmation with ceftazidime and ceftazidime-clavulanate disks, as recommended by the CLSI.

\section{RESULTS}

A total of 20,742 clean-catch urine samples were processed during a 1 year study period (April 2011 until April 2012), of which $1228(5.92 \%)$ uropathogen were isolated. We found $801 \mathrm{E}$. coli (65.2\%), 33 other Enterobacteriaceae (2.7\%), 18 non-fermentative Gram-negative bacteria (1.5\%), 321 Staphylococcus (26.1\%), 55 Streptococcus $(4.5 \%)$ by routine biochemical tests. From April 2011 to April 2012, there were 20,742 urine samples received. A total of 801 consecutive E. coli isolates from urine samples from patients attending the lab were collected. Among the above outpatients, $1053(85.7 \%)$ were females $(\max =88$ years, $\min =$ 1 months, mean $=34.7 \pm 21.8), 175(14.3 \%)$ were males $(\max =$ 89 years, $\min =3$ months, mean $=42 \pm 27.8$ ). Co-amoxiclav was the antimicrobial agent to which the highest resistance proportion $(68.6 \%)$ was observed among the tested $E$. coli urinary isolates. $E$. coli showed the high resistance rate against some of the primary drugs tested: amikacin (66\%), co-trimoxazole (65.2\%), tetracycline $(59.3 \%)$ and cephalotin (58.9\%) [Table 1]. Comparatively, it showed low resistance rate against other drugs: nitrofurantoin (14\%), chloramphenicol $(17.6 \%)$, gentamicin $(21.2 \%)$ and ceftizoxime $(21.3 \%)$. Out of the 13 antibiotics tested for E. coli isolates, the least resistance was observed to ampicillin (9.4\%). There was an evidence of a more marked resistance to co-trimoxazole and firstgeneration cephalosporins, probably due to their overuse, compared with gentamicin, ceftizoxime and nitrofurantoin.

There was no significant difference in resistance rates for all antibiotics tested in the strains from males and females. ${ }^{[8]}$

\section{DISCUSSION}

Microbial infection of the urinary tract is one of the most common infectious diseases world-wide. Today, antimicrobial resistance is increasing and antimicrobial resistance patterns varies over time and in different geographical regions, antibiotic treatment of infections should be based on local experience of sensitivity and resistance patterns. The present study provides the information about the resistance prevalence in uncomplicated UTI and allows comparison of the community in Kermanshah, Iran with other parts of the country. In this study, out of 20,742 patients from who urine samples were taken, nearly $5.92 \%$ had a UTI. This is possibly because UTI symptoms are not a reliable indicator of infection. The total growth positive rate $(5.92 \%)$ observed in this study was very lower in comparison to the finding of Saffar et al. (12.6\%) from

\begin{tabular}{lccc}
$\begin{array}{l}\text { Table 1: The susceptibility pattern of E. coli isolated } \\
\text { from UTI in outpatients }\end{array}$ \\
\hline Antibiotics & \multicolumn{3}{c}{ Susceptibility; no. (\%) of isolates } \\
\cline { 2 - 4 } & $\begin{array}{c}\text { Resistance } \\
\text { (\%) }\end{array}$ & $\begin{array}{c}\text { Intermediate } \\
\text { (\%) }\end{array}$ & $\begin{array}{c}\text { Susceptible } \\
\text { (\%) }\end{array}$ \\
\hline Cotrimoxazole & $510(65.2)$ & $67(8.7)$ & $193(25.1)$ \\
Norfloxacin & $297(40.3)$ & $80(10.9)$ & $360(48.8)$ \\
Gentamicin & $156(21.2)$ & $98(13.3)$ & $482(65.5)$ \\
Nitrofurantoin & $102(14)$ & $84(11.5)$ & $543(74.5)$ \\
Amikacin & $480(66)$ & $101(13.9)$ & $146(20.1)$ \\
Ampicillin & $66(9.4)$ & $138(19.6)$ & $500(71)$ \\
Nalidixic acid & $355(51.4)$ & $56(8.1)$ & $279(40.4)$ \\
Chloramphenicol & $120(17.6)$ & $26(3.8)$ & $537(78.6)$ \\
Ceftizoxim & $126(21.3)$ & $90(15.2)$ & $376(63.5)$ \\
Tetracycline & $317(59.3)$ & $52(9.7)$ & $166(31)$ \\
Cefalexin & $242(45.9)$ & $96(18.2)$ & $189(35.9)$ \\
Coamoxicylin & $306(68.6)$ & $75(16.8)$ & $65(14.6)$ \\
Cephalotin & $136(58.9)$ & $41(17.7)$ & $54(23.4)$ \\
\hline
\end{tabular}

E. coli $=$ Escherichia coli, $\mathrm{UTI}=$ Urinary tract infections 
$\operatorname{Iran}{ }^{[8]}$. However this was in agreement with other study conducted by Chhetri et al. (21.8\%), Rai et al. (28.6\%) and Kumari et al. $(25.7 \%)$ from Nepal. ${ }^{[9-11]}$ The majority of UTIs, Gram-negative organisms, especially $E$. coli was found to be the most frequent agent, as expected and the high resistance rate to antimicrobials. ${ }^{[12-14]}$

In the present study, as in previous report from Iran by Kashef et al., UTI occurred more in females than in males, which $85.2 \%$ of all patients were female. ${ }^{[14]}$

This is as a result of shorter and wider urethra in women, therefore bacteria have less distance to travel and get to the bladder to cause infection. Furthermore the female urethra is contaminated from the vagina and its proximity to the perineum and anus, so gut bacteria are likely to cause urinary infection. ${ }^{[13]}$ Almost $59-69 \%$ of the UPECs were resistant to co-amoxiclave, amikacin, co-trimoxazole, tetracycline and cephalotin and nearly half of them were resistant to nalidixic acid and cephalexin, indicating that empiric treatment with these antibiotics is inadequate for UTI in out-patients in our region. Resistance rats to ampicillin is $(9.4 \%)$ in the present study. This result, lower than that obtained by other studies (84-100\%), may be to un-usage of ampicillin in empirical treatment in recent years in our area ${ }^{[14]}$ It seems un-usage of antibiotic in special period of time can be cause of the decrease in resistance of bacteria against antibiotic. In our study, amikacin and co-trimoxasole are not good choice for the empirical treatment of UTI in our region. Many of the factors may contributed to such high rates of resistance to above antimicrobial agents explained by the easy access to drugs even without a prescription or inadequate dosage of these antibiotics used during selfmedication. Hence empirical therapy with these antibiotics seems inadequate and should be avoided. Unfortunately, antibiotics co-amoxiclave of new antibiotics that expensively in price, but high used in our region. The resistance $68.6 \%$ for antibiotics were not unexpected. In our study showed that these isolates were low resistant to Ampicilin, nitroforantoein, cloramphenicol, gentamicin and ceftizoxim. These antibiotics effective for the treatment of infections caused by UPEC isolated.

\section{CONCLUSION}

this study confirms that $E$. coli is still the most common uropathogen isolated. Augmentin and amikacin are not as a first choice for treatment of UTI in Kermanshah area. Ampicillin and nitrofurantoin may be considered as a first choice empiric agent in outpatients. Antibiotic resistance pattern of organisms changing rapidly over a short period therefore these finding also reinforce the need for ongoing investigation to show trends in antibiotic resistance, which can help effective empiric therapies.

\section{ACKNOWLEDGMENTS}

The authors would like to acknowledge Kermanshah University of Medical Sciences. The study was financially supported by the Kermanshah University of Medical Sciences for grant 92311.

\section{REFERENCES}

1. Hanley J, Branford I, Gugnani HC, Wilkinson C, Uhrin T. Urinary bacterial pathogens and their antimicrobial susceptibility profile for the years 20052007 in St Kitts. West Indian Med J 2009;58:571-4.

2. Raz R, Stamm W. Optimal treatment of UTI in postmenopausal women. J Am Med Dir Assoc 2000;1:172-4.

3. Kurtaran B, Candevir A, Tasova Y, Kibar F, Inal AS, Komur S, et al. Antibiotic resistance in community-acquired urinary tract infections: Prevalence and risk factors. Med Sci Monit 2010;16:CR246-51.

4. Santo E, Salvador MM, Marin JM. Multidrug-resistant urinary tract isolates of escherichia coli from Ribeirão Preto, São Paulo, Brazil. Braz J Infect Dis 2007;11:575-8.

5. Alizadeh Taheri $P$, Navabi B, Shariat M. Neonatal urinary tract infection: Clinical response to empirical therapy versus in vitro susceptibility at Bahrami Children's Hospital- Neonatal Ward: 2001-2010. Acta Med Iran 2012;50:348-52.

6. Clinical and Laboratory Standards Institute. M7-A7. Dilution Antimicrobial Susceptibility Tests for Bacteria that Grow Aerobically. Wayne, PA: CLSI; 2007.

7. Bahadin J, Teo SS, Mathew S. Aetiology of community-acquired urinary tract infection and antimicrobial susceptibility patterns of uropathogens isolated. Singapore Med J 2011;52:415-20.

8. Saffar MJ, Enayti AA, Abdolla IA, Razai MS, Saffar H. Antibacterial susceptibility of uropathogens in 3 hospitals, Sari, Islamic Republic of Iran, 2002-2003. East Mediterr Health J. 2008;14:556-63.

9. Chhetri PK, Rai SK, Pathak UN, Thapa JB, Devkota KC, Shrestha BO, Shrestha RR. Retrospective study on urinary tract infection at Nepal Medical College Teaching Hospital. Nepal Med Coll J. 2001;3:83-5.

10. Rai GK, Upreti HC, Rai SK, Shah KP, Shrestha RM. Causative agents of urinary tract infections in children and their antibiotic sensitivity pattern: a hospital based studyNepal Med Coll J 2008;10:86-90.

11. Kumari N, Ghimire G, Magar JK, Mohapatra TM, Rai Antibiogram pattern of isolates from UTI cases in Eastern part of Nepal. Nepal Med Coll J 2005;7:116-8.

12. Yilmaz N, Agus N, Yurtsever SG, Pullukcu H, Gulay Z, Coskuner A, et al. Prevalence and antimicrobial susceptibility of Escherichia coli in outpatient urinary isolates in Izmir, Turkey. Med Sci Monit 2009;15:161-5.

13. Acharya A, Gautam R, Subedee L. Uropathogens and their antimicrobial susceptibility pattern in Bharatpur, Nepal. Nepal Med Coll J 2011;13:30-3.

14. Kashef N, Djavid GE, Shahbazi S. Antimicrobial susceptibility patterns of community-acquired uropathogens in Tehran, Iran. J Infect Dev Ctries 2010;4:202-6.

How to cite this article: Jalilian S, Farahani A, Mohajeri P. Antibiotic resistance in uropathogenic Escherichia coli isolated from urinary tract infections out-patients in Kermanshah. Int J Med Public Health 2014;4:75-7.

Source of Support: Kermanshah University of Medical Sciences. Conflict of Interest: None declared. 